\title{
Temperature Effects on the Fatigue of Highly filled PMMA
}

\author{
Okite Obakponovwe and Gordon Williams \\ Department of Mechanical Engineering \\ Imperial College London \\ SW7 2AZ, United Kingdom
}

\begin{abstract}
The modulus and fracture toughness of an ATH-filled PMMA composite are determined as a function of temperature. The modulus can be modelled as a series addition of the two phases, giving a decreasing modulus with temperature tending to zero at $110^{\circ} \mathrm{C}$. The $K_{c}$ value remains constant. Fatigue crack growth data in the form of $d a / d N$ versus $K$ were obtained as a function of temperature and modelled using the Paris Law. The power index remained constant at seven, but the coefficient had a maximum at $50^{\circ} \mathrm{C}$. It is suggested that this arises from microcracks generated by interparticle thermal stresses which are shown to have a similar maximum.
\end{abstract}

Keywords: Solid surface, particulate composites, debonding, fracture, fatigue, failure mechanism.

\section{Introduction}

Particulate filled polymers are widely used as solid surfaces in kitchens and bathrooms, as well as in the fabrication of sinks and other bathroom vanities. The addition of the second phase (ATH filler) increases the material's stiffness and aesthetic properties (texture and appearance). Generally the mechanical performance is good, but in their main applications, the materials are often subjected to thermal cycling. This occurs around cut-outs in which hot plates are located, and in sinks subjected to alternating flows of hot and cold water. In both cases, the temperature cycles give rise to stress cycles and this in turn can lead to the propagation of fatigue cracks. It is not clear, a priori, if the material is damaged by temperature cycling or if the crack growth is simply due to the stress generated at various temperatures. To clarify this issue, some conventional fatigue crack propagation tests were performed to measure the crack propagation rate, $d a / d N$, for a range of constant temperatures which covered the operating range [1].

\section{Materials and Experiments}

The material used is a lightly cross-linked PMMA filled with about $40 \%$ by volume of Alumina Trihydrate (ATH) particles in the $2-10 \mu \mathrm{m}$ size range. The $12.5 \mathrm{~mm}$ thick sheets is made by a continuous casting process, with slow cooling to minimise warping and residual stresses. Preliminary tests were performed by measuring elastic modulus in the $0-100^{\circ} \mathrm{C}$ temperature range in both simple tension and three point bend testing. The results are presented in figure 1. The PMMA matrix has a modulus of about $3 \mathrm{GPa}$ at $20^{\circ} \mathrm{C}$ and a glass transition 
temperature of $110^{\circ} \mathrm{C}$ with a linear dependence so the behaviour presented in figure 1 is consistent with a lower bound series interaction and can be fitted with:

$$
E=18\left(\frac{110-T}{188-T}\right) G P a
$$

and this line is also shown in figure 1. This assumes that the modulus of ATH is independent of temperature.

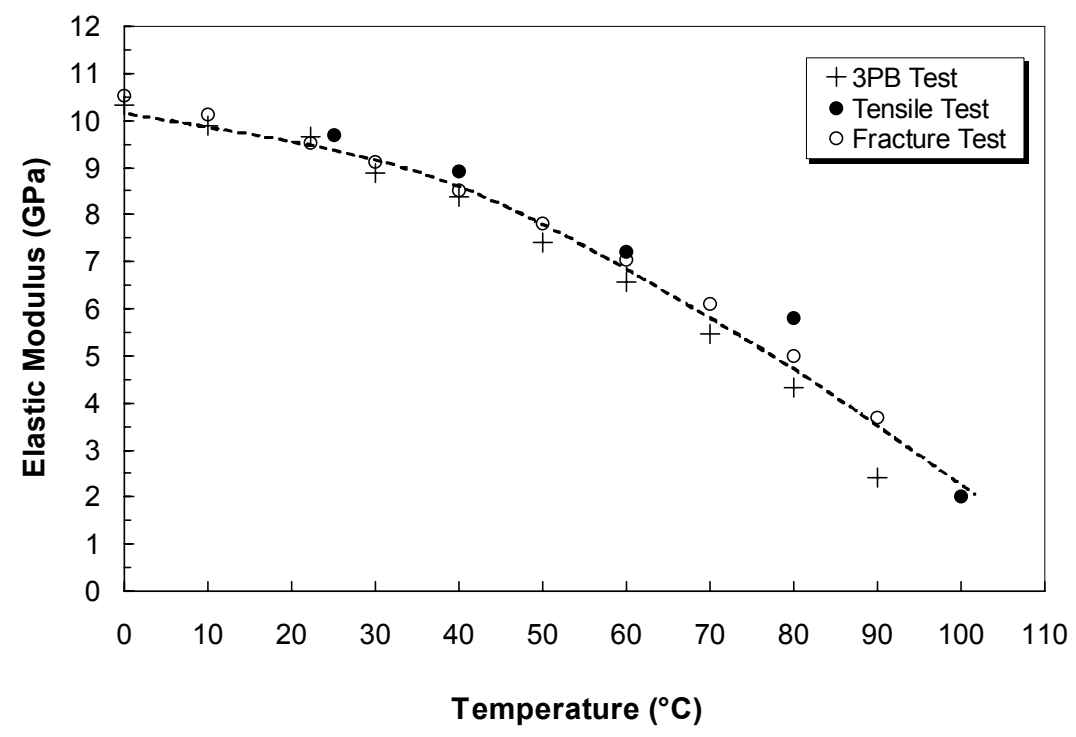

Figure 1: Elastic modulus against temperature comparing results from 3-Point bend, tensile and fracture tests.

The low rate fracture toughness was evaluated using the ISO standard for deducing $K_{c}$ and $G_{c}$ for polymers [2]. The compact tension (CT) geometry shown in figure 2 was convenient since the thickness of the sheet could be used as the thickness of the CT specimen. 


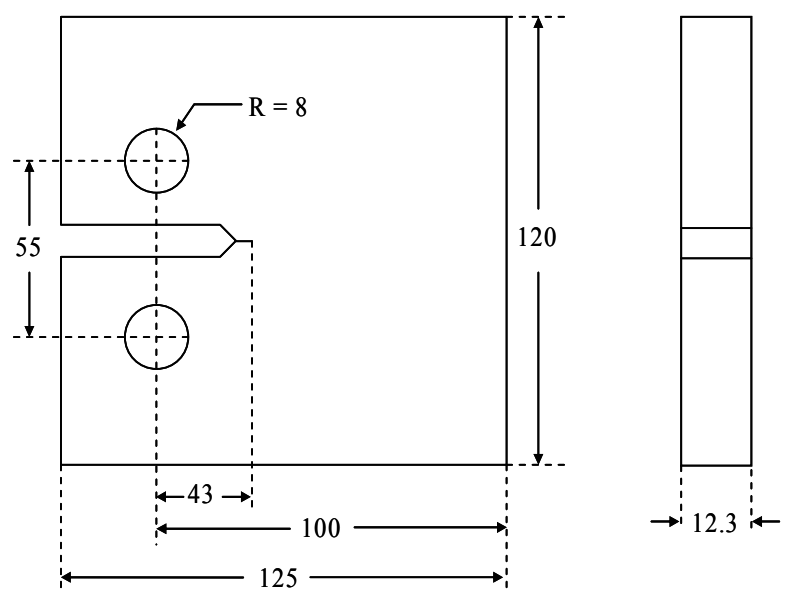

Figure 2: CT specimen configuration (all dimensions in $\mathrm{mm}$ ).

The tests were constructed in a screw-driven Instron machine at $1 \mathrm{~mm} / \mathrm{min}$, and the values for $K_{c}$ at initiation are given by:

$$
K_{I C}=f_{(a / W)} \frac{F}{b \sqrt{W}}
$$

where $F$ is the load, $f$ a calibration factor, $b$ the thickness, and $W$ the width. The temperature was controlled by a cabinet enclosing the specimen, and was determined by inserting thermocouples in small holes drilled to the centre of the sheet. Figure 3 shows the results for $K_{I C}$ as a function of temperature and it remains remarkably constant at $2 \mathrm{MPa} \sqrt{\mathrm{m}}$ over this range. The load deflection curves were linear and $G_{I C}$ was found from the area under the curve, i.e. the energy. $E, K_{I C}$ and $G_{I C}$ are related via,

$$
E=\frac{K_{I C}^{2}}{G_{I C}}
$$

So $E$ could be found and these values are also shown in figure 1 . The fact that they are about $15 \%$ lower than the other methods is common, and reflects the difficulty in correcting for machine stiffness. Since $E$ decreases and $K_{I C}$ is constant, $G_{I C}$ increases with temperature from $402 \mathrm{~J} / \mathrm{m}^{2}$ at $20^{\circ} \mathrm{C}$ to $1000 \mathrm{~J} / \mathrm{m}^{2}$ at $80^{\circ} \mathrm{C}$. The broken line in figure 3 is that for PMMA [3] and shows a decrease in $K_{I C}$ and the equivalent values of $\mathrm{G}_{I C}$ are constant at about $1000 \mathrm{~J} / \mathrm{m}^{2}$. 


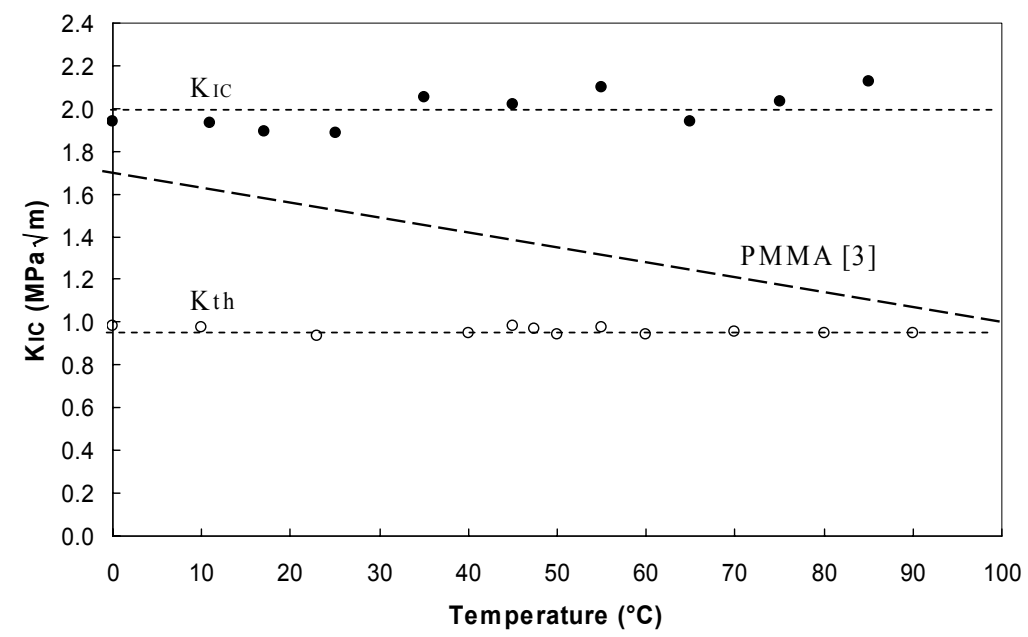

Figure 3: $K_{I C}$ and $K_{t h}$ as a function of temperature for the filled polymer.

\section{Fatigue Tests}

The fatigue tests were performed according to ISO protocol [4], and employed the CT specimen and temperature cabinet. The tests were at constant load so that $K_{C}$ and hence $d a / d N$, could be determined during crack growth. The growth was measured using thin metal foils and a Fractomat unit. Curves of $a$ versus $N$ were determined and hence $d a / d N$ as a function of $K$ was found. The cycling was to zero load, i.e. an " $R$ " ratio of zero. Figure 4 shows three typical curves of $d a / d N$ versus $K$ on a log-linear basis and lines drawn to fit the Paris Law,

$$
\frac{d a}{d N}=A \cdot K^{m}
$$

There are well defined threshold values $K_{t h}$ (also shown in figure 3), and are constant at about $1 \mathrm{MPa} \sqrt{\mathrm{m}}$. The slope $m$ is also constant at about 7, but it is noticeable that that data at $50^{\circ} \mathrm{C}$ is higher than at 23 and $80^{\circ} \mathrm{C}$. This is reflected in the values of $A$ which are shown in figure 5, and have a distinct $A$ maximum at about $50^{\circ} \mathrm{C}$. This is a somewhat unexpected result since $K_{I C}, K_{t h}$ and $m$ are constant with temperature. 


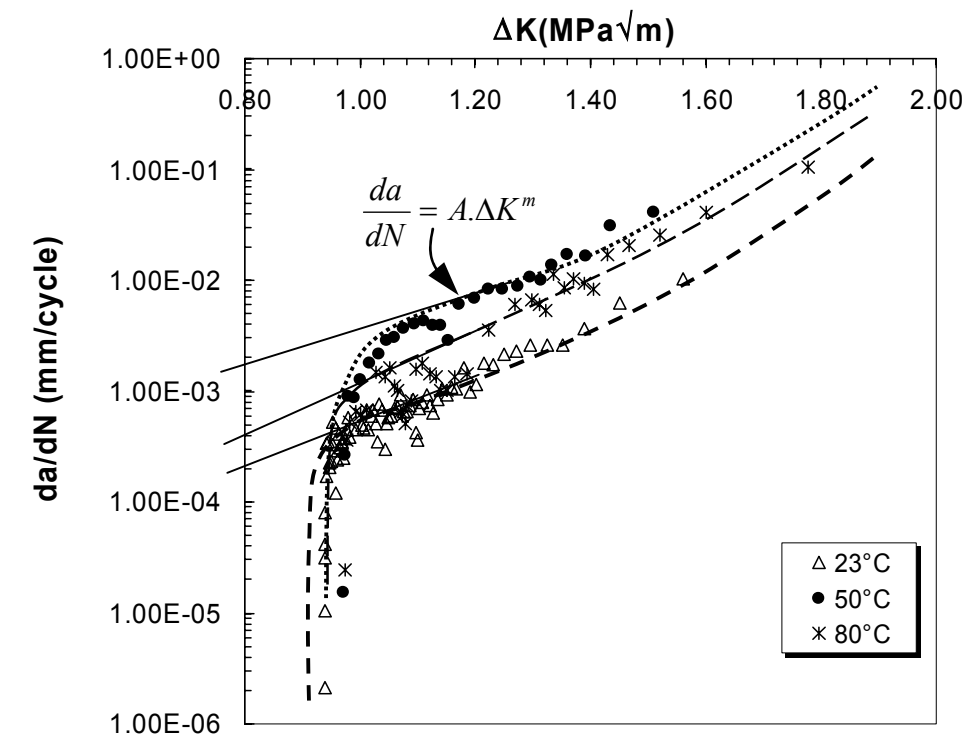

Figure 4: The effect of temperature on the fatigue growth rate curves.

\section{Discussion}

A possible explanation of the peak in $A$ is the generation of interparticle stresses with increasing temperature. There is a very large difference in the thermal expansion coefficient $\alpha$ between the matrix and the particles, and a measure of this thermal stress is given by,

$$
\sigma_{T}=E \Delta \alpha \cdot(T-\bar{T})
$$

where $\Delta \alpha$ is the difference in $\alpha$ between the two phases, $\bar{T}$ is the temperature at which there is no thermal stress. Substituting for $E$ from eq. 1, and using $\Delta \alpha=0.9 \times 10^{-6}{ }^{\circ} \mathrm{C}^{-1}$ we have,

$$
\sigma_{T}=1.6\left(\frac{110-T}{188-T}\right)(T-\bar{T}) M P a
$$

$\bar{T}$ is unknown but we could expect $\sigma_{T}$ to be finite at ambient conditions because of the residual effects of processing. If we use $\bar{T}=-34^{\circ} \mathrm{C}$, there is a peak in $\sigma_{T}$ at about $50^{\circ} \mathrm{C}$, and it gives $\sigma_{T} \approx 50 \mathrm{MPa}$ at $20^{\circ} \mathrm{C}$. Equation 4 is shown plotted in fig. 5, and clearly $A$ correlates with $\sigma_{T}$. The fracture surface is rough on a small scale and a mechanism involving microcracking around particles is a possible explanation. 


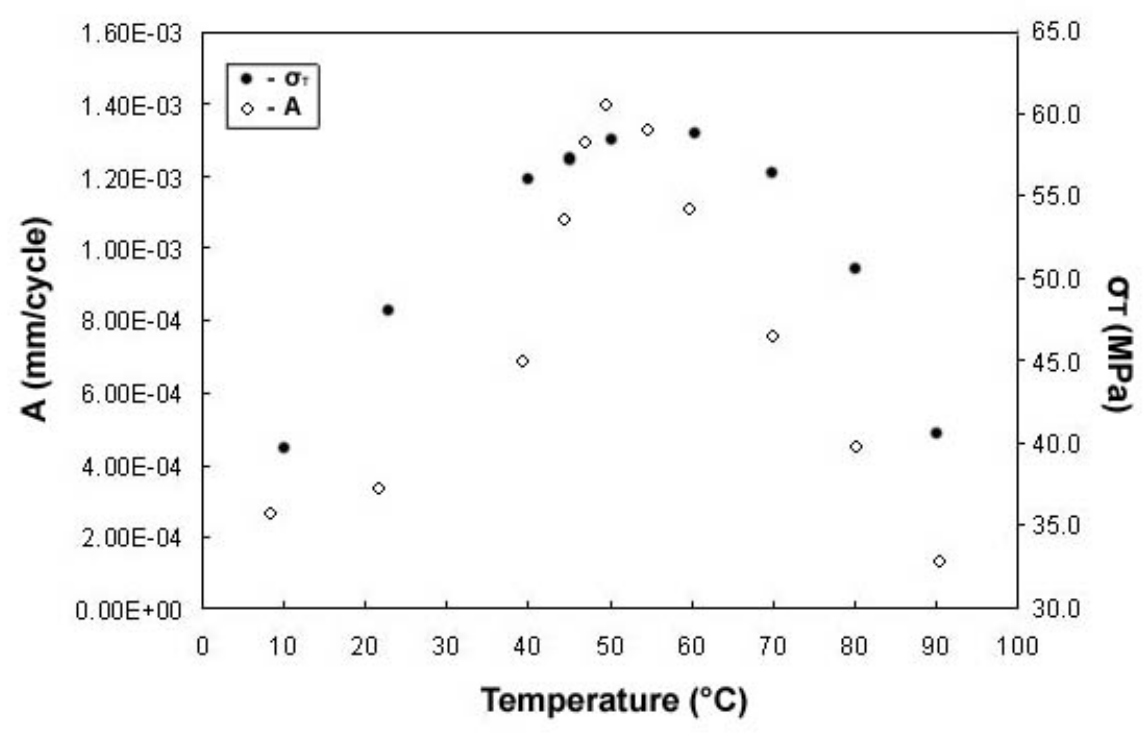

Figure 5: $A$ and thermal stress as a function of temperature

Detailed modelling via the Paris Law is difficult because $A$ has no physical significance and the effect will be modelled more usefully via a damage zone model as given in [3]. A detailed analysis is given in [5].

\section{References}

[1] O. Obakponovwe, PhD Thesis, "Temperature Effects in Fatigue of Alumina-Filled PMMA”, Imperial College London, Aug. 2003.

[2] ISO/DIS 13586, "Plastics - Determination of Fracture Toughness $\left(G_{\mathrm{C}}\right.$ and $\left.K_{\mathrm{C}}\right)-$ LEFM approach.

[3] J. G. Williams, "Fracture Mechanics of Polymers," Ellis Horwood Ltd (1987).

[4] ESIS TC4, "A Protocol for Tension-tension Fatigue Crack Propagation Testing of Plastics", Technical Committee on Polymers and Composites, 3 (2000).

[5] O. Obakponovwe \& J. G. Williams, to be published

\section{Acknowledgements}

The authors wish to thank DuPont for the supply of the material and financial support. 